\title{
Calcaneal Lengthening Using Bone Substitute Graft for Neurological Flatfoot Treatment
}

\author{
Bruno Casaccio, Dra Silvia Reciniello and Mario Lampropulos* \\ School of Medicine, Argentina \\ *Corresponding author: Mario Lampropulos, School of Medicine, Universidad de Buenos Aires, Ministry of Health and Social Services, Argentina
}

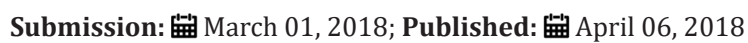

\begin{abstract}
Flatfoot with hindfoot valgus is a common problem in neurological patients. It characterized by the loss of the longitudinal arch, pain, ulceration and dermal plantar hyperkeratosis, shortening of the Achilles tendon and failure in the use of orthoses. The objective of our job is to show the results of the neurological flatfoot treatment with calcaneal lengthening using Bone Substitute graft (Pro-Dense ${ }^{\circledR}$ ). Material and methods: A retrospective study was carried out in which we included all patients who underwent surgery for neurological symptomatic flatfoot. Measures were made of the Talo-Calcaneal angle, Talo-1MT y Calcaneal Pitch. It has been also analyze the postoperative clinic results. Results: 17 patients (27 feet). Astragalus-Calcaneo angle preoperative $26.07^{\circ}$ and postoperative de $21.22^{\circ}$. Talo- $1 \mathrm{MT}$ angle $27.04^{\circ}$ preoperative y $13.22^{\circ}$ postoperative. Calcaneal Pitch preoperative angle $12.63^{\circ}$ and postoperative $21.37^{\circ}$. It was observed a statistically significative change. In all cases we obtain painless feet, with the disappearance of the plantar hyperkeratosis, correction of the hindfoot valgus and longitudinal arch formation. Conclusions: The calcaneal lengthening with Bone Substitute graft technique is an alternative for the Neurological flatfoot treatment. With good results, corrections of the angular deformities and low rate of complications.
\end{abstract}

\section{Introduction}

Flatfoot is a frequent pathology in neurological patients; it can be seen in cerebral palsy, genetic syndrome, myelomeningocele patients, as a result of a overcorrection of club foot [1,2]. It characterizes by the loss of the longitudinal arch, pain, ulceration and dermal plantar hyperkeratosis, shortening of the Achilles tendon and failure in the use of orthoses. In 1975, Evans described the calcaneal lengthening technique for the treatment of flatfoot in children [3] later on, Mosca in 1995, modify this technique and prove its utility in patients with neurological diseases [4].

The most well-known techniques use Autologous iliac crest bone graft or tricortical cadaveric allograft for the correction and the elongation of calcaneal. The authors are not aware of the existence of publications where they show the results of using Bone Substitute to perform the correction in the technique of Evans/ Mosca [5,6].

The aim is to show the results of neurological flatfoot with calcaneal lengthening using Bone Substitute (Pro-Dense $®$ ) treated in our institution.

\section{Materials and Methods}

A retrospective study was carried out in which we included all patients who underwent surgery in our institution for correction of neurological symptomatic flatfoot with calcaneal lengthening technique using bone substitute graft since 2007 to June 2016 with a minimum follow up of 1 year.

Patients that were treated with other techniques have been excluded of the study. (Tenotomies, Capsular releases, Triple arthrodesis). All interventions had been carried out by the same Neuro-orthopedic surgeon (ML). Radiography controls were made immediately in the postoperative and 4 weeks later to evaluate results and consolidation.

We made angle measures in Talo-Calcaneal AP radiograph, Talo-1MT angle and Calcaneal Pitch in lateral radiography and have compared the preoperative values and the postoperative values to evaluate the correction of the deformity. In addition, postoperative angles were compared with normal theoretical values, being these for Talo-Calcaneal angle 20.0 \pm 5.063 , Talo-1MT angle $13.5 \pm 6.582$ and Calcaneal Pitch 22.5 \pm 7.595 [7].

We also evaluated the postoperative clinical results according to Mosca's criteria [4], being a successful outcome when valgus deformity of the hindfoot had been corrected, a longitudinal arch had been created, and the prominence of the talar head had been eliminated; the pain and callus under the head of the talus had been eradicated; the ulceration had not recurred; and tolerance of the brace and shoes had improved. 


\section{Surgical technique}

The patients in dorsal decubitus, made an incision in the lateral face of the hindfoot at sinus tarsi level. The peroneus longus and brevis tendons were identified and we performed a tenoplasty. An Osteotomy was performed with oscillating saw to $1.5 \mathrm{~cm}$ from the distal articular facet of the Calcaneus. The osteotomy was completed with laminate chisel if necessary. Then a spreader was placed and we proceeded to place the graft of substitute bone previously molded, as explained below.

We use Bone Substitute as graft (Pro-Dense $®$ ), it is a synthetic compound of calcium sulfate. This product has been used in orthopedic surgery for fulfilling posttraumatic cavities and oncological resections. In our technique, we use the product in solid form for realizing the correction and like graft. For achieving that it is recommended to prepare the product by its instructions in a sterile container, take control until you get wedges made by hand by an assistant surgeon, then it can be adjust to the right measure for filling the osteotomy and achieve the correction desired (Figure 1).

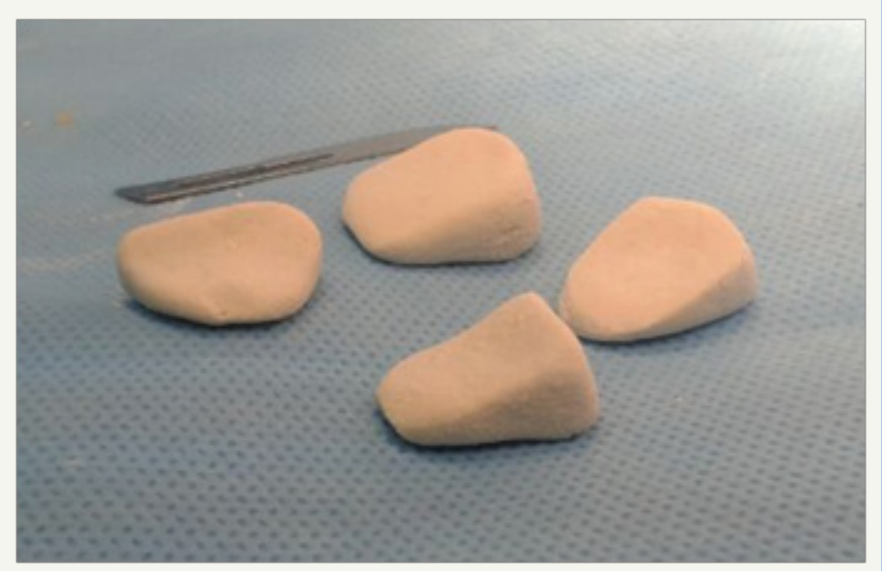

Figure 1

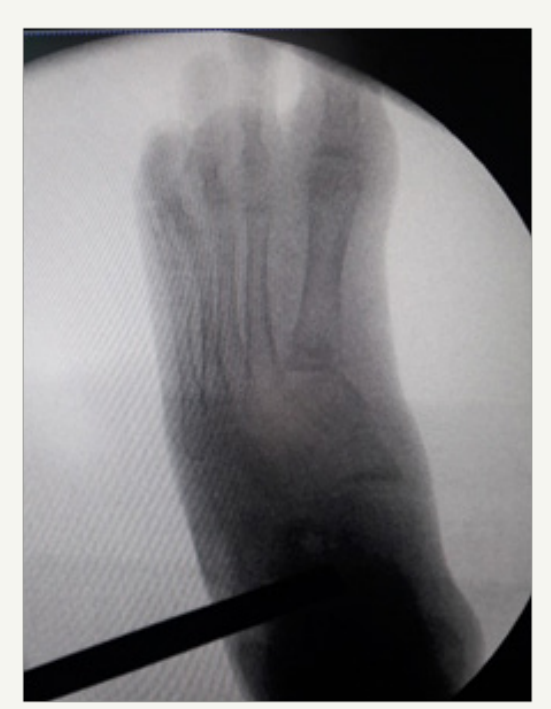

Figure 2
Clinical and Radiographic correction is controlled intraoperatively. The Osteotomy is fixed with rhombic compression staples (Charlotte ${ }^{\circledR}$ Wright). The incision is closed with absorbable suture in planes. If it is necessary, Achilles tenoplasty is performed at the same surgical time (Figure 2).

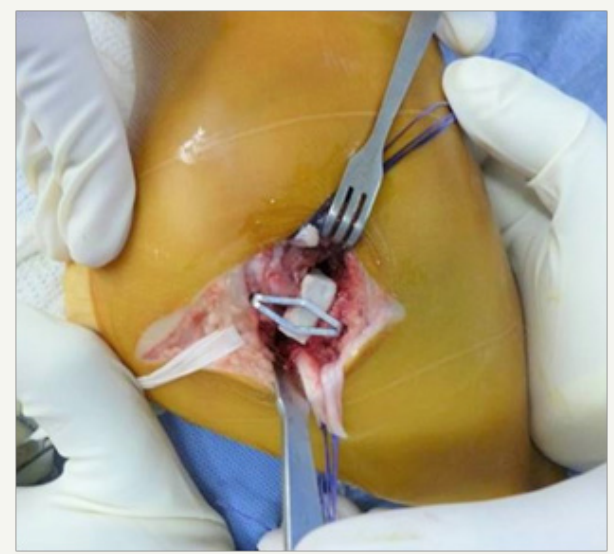

Figure 3.

Patients were immobilized with plaster short boot for 4 weeks. Plaster windows were made to perform the cures and it was only change (cast) in case of complications or break of it (Figure 3).

After the fourth week the immobilization was changed to a AFO orthosis and the patient started with rehabilitation. Weight load was not allowed until the fifth week.

\section{Results}

17 patients (27 feet), 10 male patients (55\%) and 7 female patients (45\%). The average age was 12,7 years with a range between 4 and 27 years. The follow up average was of 27 months with a range between 60 and 13 months.

Of the total of patients, $12(70 \%)$ has diagnosis of Cerebral Palsy, 1 of them associated to Neurofibromatosis and 5 (30\%) patients with diagnosis of Myelomeningocele.

All patients had symptomatic flatfoot (walking pain, difficult for long walking distance and difficult for the orthosis use or skin injuries with footwear and orthosis).

From the total of the patient's, 16 were community ambulators (12 patients) or home ambulators ( 4 patients) and 1 of the patients was not ambulator.

1 patient presented previous treatment of his pathology in another institution where surgery correction by multiple tenoplasties was attempted with 3 years of evolution and relapse of deformity.

In all cases our patients got painless feet, with the disappearance of the plantar hyperkeratosis, a correction of the hindfoot valgus and appearance of the longitudinal arch. Patients, who were previously ambulators, could return to their normal activities and no problems for footwear and the use of orthosis were found. 
These were evaluated as satisfactory clinical results according to the criteria of Mosca previously cited.

A statistical analysis was performed of the obtained results with a range test with sign of Wilcoxon which is a non-parametric test to compare the midrange of two related samples (or not) and determine if there are differences between them. Used as an alternative to the t-Student test when the normality of these samples cannot be assumed. This test also allows you to assess whether a sample comes from a theoretical population, replacing the t-Student test to evaluate if the sample mean is equal to the population mean $\mu$. Test and graphics were made with the software Ri386 3.4.0.

The results of the comparison of correction angles pre and postoperative can be seen in Table 1 . The measure of the angle TaloCalcaneus preoperative was of $26.07^{\circ}$ and postoperative of $21.22^{\circ}$ (Graphic 1), for the Talo-1MT angle were of 27.04 preoperative and $13.22^{\circ}$ postoperative (Graphic 2) and for the Calcaneal Pitch angle preoperative $12.63^{\circ}$ and postoperative $21.37^{\circ}$ (Graphic 3).

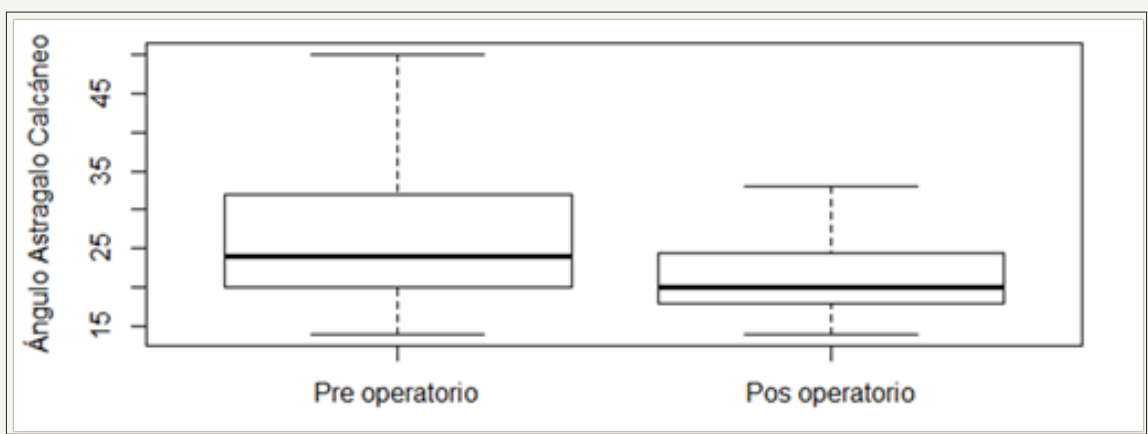

Graphic 1: Talo-calcaneal angle boxplot according to measurement moment.

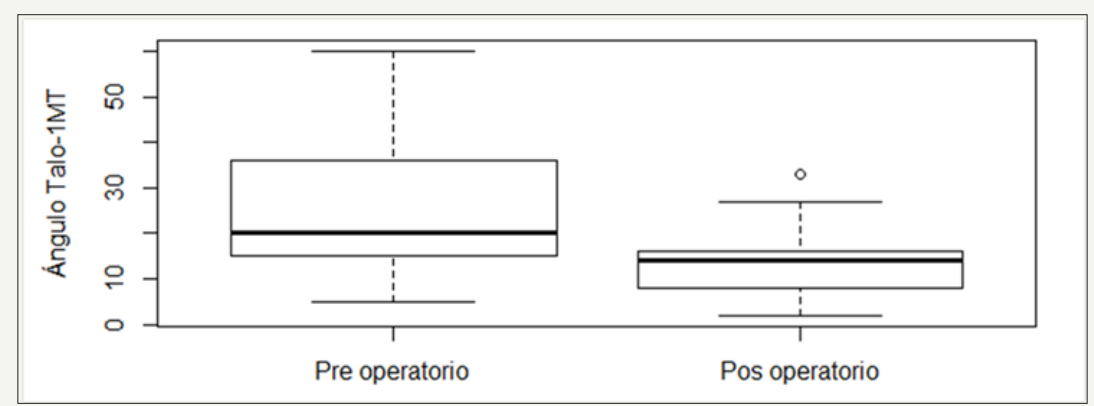

Graphic 2: Talo-1MT angle boxplot according to measurement moment.

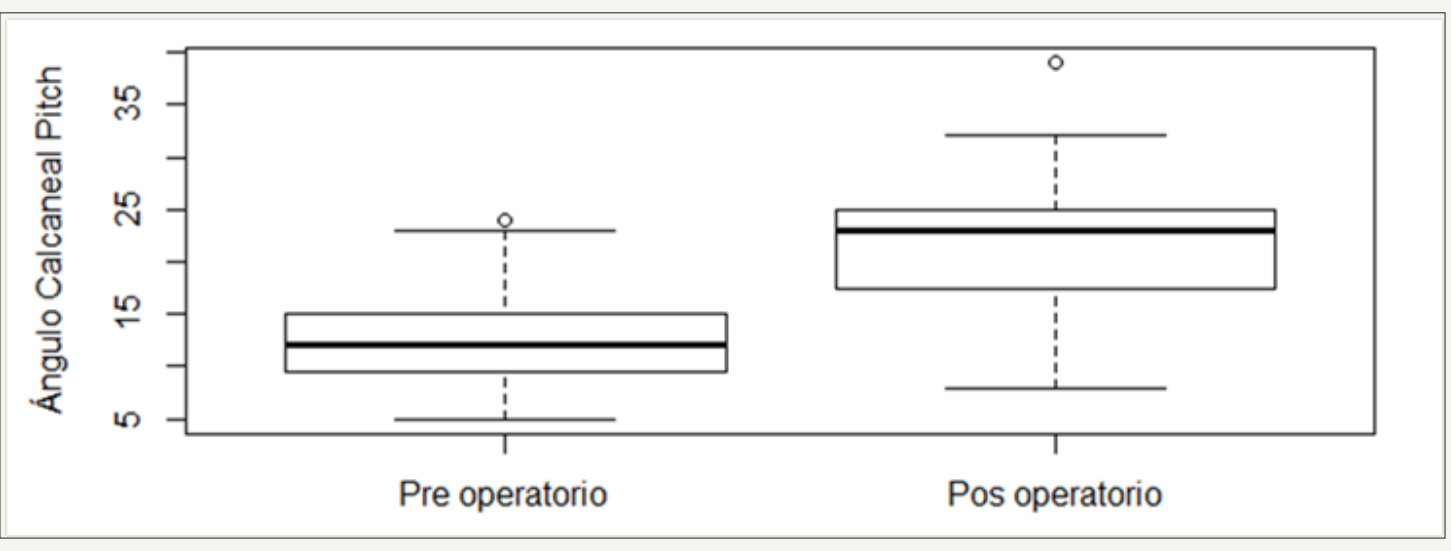

Graphic 3: Calcaneal Pitch angle boxplot according to measurement moment.

Is observed a statistically significant change in terms of the angles pre and post operatives (all the p-values are minors to $\alpha=0,05$ ), observing that the angles post-operatives approach the normal values of correction.

To test this, it was evaluated then if the values post-operatives obtained have a tendency central statistically equal to the theoretical values, results are presented in Table 2 . It was observed that the angles post-operatives obtained have a central tendency statistically equal to the expected theoretical values (all the p-values are greater than $\alpha=0.05$ ). 
Table 1: Comparison of pre and post-operative correction angles.

\begin{tabular}{|c|c|c|c|}
\hline Angle & \multicolumn{2}{|c|}{ Media \pm SD $\left({ }^{\circ}\right)$} & p-valor1 \\
\hline & Pre0p & Pos0p & 0.01755 \\
\hline Talo-calcaneal & $26.07 \pm 8.935$ & $21.22 \pm 4.414$ & 0.00009 \\
\hline Talo-1MT & $27.04 \pm 16.985$ & $13.22 \pm 7.904$ & 0.00007 \\
\hline Calcaneal Pitch & $12.63 \pm 5.248$ & $21.37 \pm 7.060$ & \\
\hline
\end{tabular}

Table 2: Comparison of postoperative correction angle with expected theoretical values.

\begin{tabular}{|c|c|c|c|}
\hline Angle & \multicolumn{2}{|c|}{ Media \pm SD $\left(^{\circ}\right)$} & p-valor2 \\
\hline & Valor Pos0p & Valor Teórico & 0.1843 \\
\hline Talo-Calcaneal & $21.22 \pm 4.414$ & $20.0 \pm 5.063$ & 0.5966 \\
\hline Talo-1MT & $13.22 \pm 7.904$ & $13.5 \pm 6.582$ & 0.4627 \\
\hline Calcaneal Pitch & $21.37 \pm 7.060$ & $22.5 \pm 7.595$ & \\
\hline
\end{tabular}

Table 3: Comparison of preoperative correction angle according to the type of ambulation

\begin{tabular}{|c|c|c|c|}
\hline Preoperative Angle & \multicolumn{2}{|c|}{ Media \pm SD $\left(^{\circ}\right)$} & p-valor3 \\
\hline & Community Ambulators $(\mathbf{n}=\mathbf{1 9})$ & Home Ambulators $(\mathbf{n}=\mathbf{6})$ & 0.6316 \\
\hline Talo-Calcaneal & $27.52 \pm 9.269$ & $25.16 \pm 6.911$ & 0.2924 \\
\hline Talo-1MT & $24.52 \pm 16.584$ & $35.66 \pm 19.866$ & 0.2125 \\
\hline Calcaneal Pitch & $13.63 \pm 5.539$ & $10.33 \pm 4.457$ & \\
\hline
\end{tabular}

There was also a comparison of measuring angles preoperative according to ambulation status (Community or domiciliary) of the patients (Table 3). There was not a statistically different central trend for angles pre-operatives obtained according to the type of patient ambulation (all the $\mathrm{p}$-values are greater than $\alpha=0.05$ ). No relationship was found between the degree of deformity and the capacity of ambulation of patients.

The Radiographic consolidation of the osteotomy was achieved in all cases between 8 and 10 weeks postoperative. We considered consolidation as the disappearance of the osteotomy line between the graft and the host bone.

It was found in the Postoperative follow up 2 complications. 1 patient presented hypersensitivity to the level of the scar, that required treatment of scar in rehabilitation for 3 months. 1 patient presented as a complication dehiscence the surgical wound at 6 weeks postoperative, it required 2 surgical toilettes with withdrawal of material osteosynthesis antibiotic therapy and subsequent placement of continuous suction system. Healing with good evolution and resolution was obtained; the patient was doing their usual activities at 6 months after intervention.

\section{Discussion}

The bibliography about calcaneal lengthening for the treatment of neurological flatfoot with Bone Substitute is limited $[5,6]$. Noritake et al. [7] in his studies it presents 16 patients (27 feet) with cerebral palsy treated with the technique described by Mosca with Autologous iliac crest bone graft. Report that $74 \%$ of satisfactory results and $77.8 \%$ of favorable radiograph results. A correction average of $18.5^{\circ}$ in the Talo- $1 \mathrm{MT}$ angle of the lateral radiograph and $8.8^{\circ}$ average of correction of the Calcaneal Pitch. This results are comparable to the ones made in our institution were we present a correction average of the Talo- $1 \mathrm{MT}$ angle of $27^{\circ}$ and of $12.6^{\circ}$ in the Calcaneal Pitch. In relation to the results in AP radiographs, they use as measure the Talo-1MT angle and the coverage talonavicular angle, while in our studio we used the Talo-Calcáneo angle. Conclude in this study that calcaneal elongation technique describe by Mosca as useful for the flatfoot treatment in neurological patients.

In their study Rhodes et al. [8] carried out a comparison between calcaneal lengthening with Evans technique with cadaveric allograft and bovine xenograft. They presented 36 patients (63 feet), 20 patients ( 34 feet) treated with cadaveric allograft and 16 patients (29 feet) with bovine xenograft. In both cases they present a radiographic correction of the flatfoot similar, with an advantage to the xenograft bovine, with a improvement in the Calcaneal Pitch of $3.8^{\circ}$ with allograft and of $6.1^{\circ}$ with xenograft and the lateral Talo-1MT of $8.1^{\circ}$ and $14.5^{\circ}$. Regarding to the consolidation of the osteotomy radiograph, the average time until it was achieved the incorporation, in this work it was considered as difficulty for discerning the placement of the graft; it was of 11 months for the allograft and 30 months for the xenograft. We believe that the difference in time of radiographic consolidation is as a result of the diferent parameters taking into consideration. We focused our criterion in those needed to allow patients to stand and return to usual activities.

As to the utilization of Bone Substitute and its potential adverse effects and complications postoperative, by no founding similar studies to ours, we have compared the results with studies in which the same compounds were used for orthopedic surgeries with different techniques. Kumar et al. [9] present their results using Bone Substitute for bony defects in benign tumor lesions 
and chronic osteomyelitis, it was report as the only complication the download aseptic auto limited in 8 patients out of 15 totals; Evaniew et al. [10] report the use for primary bone tumors injuries and have not found any problem in his patients; Hung et al. [11] in his studies of using Bone Substitute for Hand enchromas treatment, it was reported as the most common complication, superficial erythema presence in 1 patient and aseptic secretion in the other. These three studies present similar complications as the ones found in our study, with the difference of being self-limited and aseptic. In regard to the percentage of complications, we believe that for using differents techniques and body parts, no comparison can be made.

There are some limitations in our work. In first place those that are inherent to their retrospective nature. In second place the short period of follow up, that do not let us evaluate the different complications in long-term. Also, the few number of patients, by this is that we continue raising information so we can obtain a better panorama of this surgery and its effects.

\section{Conclusion}

We believe that the Calcaneal lengthening with Bone Substitute graft technique is an alternative for treatment of neurological symptomatic flatfoot. In our case study we obtain positive results, with correction of the angular deformities in comparison with the preoperative and postoperative and a tendency of equalize normal theoretical values statistically significant, with a time of consolidation of the osteotomy and minor osteointegration in comparison with the different existing techniques and Autograft technique, that nowadays is the Gold Standard. It presents a low rate of complications, good results with improvement of the symptomatology, disappearance of plantar hyperkeratosis and return to previous activities.

\section{References}

1. van Bosse HJ (2015) Syndromic Feet: Arthrogryposis and Myelomeningocele. Foot Ankle Clin N Am 20(4): 619-644.

2. Mara S Karamitopoulos, Lana Nirenstein, Brooklyn NY (2015) Neuromuscular Foot Spastic Cerebral Palsy. Foot Ankle Clin N Am 20(4): 657-668.

3. Evans D (1975) Calcaneo-valgus deformity. J Bone Joint Surg Br 57(3): 270-278.

4. Mosca VS (1995) Calcaneal lengthening for valgus deformity of the hindfoot. Results in children who had severe, symptomatic flatfoot and skewfoot. J Bone Joint Surg Am 77(4): 500-512.

5. Logroscino V Campana, Milano G, Pagano E, Barba M, Cicione C, et al. (2014) Bone substitutes in orthopaedic surgery: from basic science to clinical practice. J Mater Sci Mater Med 25(10): 2445-2461.

6. Nandi SK, Roy S, Mukherjee P, Kundu B, De DK, Basu D (2010) Orthopaedic applications of bone graft \& graft substitutes: a review. Indian J Med Res 132: 15-30.

7. Sung KH, Chung CY, Lee KM, Lee SY, Park MS (2013) Calcaneal lengthening for planovalgus foot deformity in children with spastic cerebral palsy. Clin Orthop Relat Res 471(5): 1682-1690.

8. Rhodes J, Mansour A, Frickman A, Pritchard B, Flynn K, et al. (2017) Comparison of Allograft and Bovine Xenograft in Calcaneal Lengthening Osteotomy for Flatfoot Deformity in Cerebral Palsy. J Pediatr Orthop 37(3): e202-e208.

9. Kumar CY, KBN, Menon J, Patro DK, BHB (2013) Calcium Sulfate as Bone Graft Substitute in the Treatment of Osseous Bone Defects, A Prospective Study. J Clin Diagn Res 7(12): 2926-2928.

10. Evaniew N, Tan V, Parasu N, Jurriaans E, Finlay K (2013) Use of a Calcium Sulfate-Calcium Phosphate Synthetic Bone Graft Composite in the Surgical Management of Primary Bone Tumors. Orthopedics 36(2): e216-e222.

11. Hung YW, Ko WS, Liu WH, Chow CS, Kwok YY, et al. (2015) Local review of treatment of hand enchondroma (artifcial bone substitute versus autologous bone graft) in a tertiary referral centre: 13 years' experience. Hong Kong Med J 21(3): 217-223.
Creative Commons Attribution 4.0 International License

For possible submissions Click Here

\section{Submit Article}

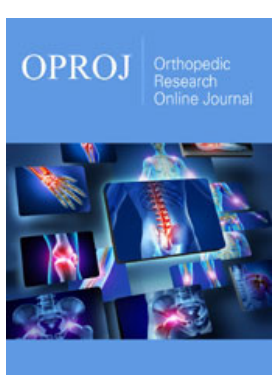

Orthopedic Research Online Journal

\section{Benefits of Publishing with us}

- High-level peer review and editorial services

- Freely accessible online immediately upon publication

- Authors retain the copyright to their work

- Licensing it under a Creative Commons license

- Visibility through different online platforms 